\title{
Early removal of asymptomatic mandibular third molar-is it safe?
}

\author{
Xiao F. Ling*, Tee L. Heng, Ahmad F. Bin Mohamad
}

\section{Abstract}

Objective: The purpose of this study was to compare the incidence of complications between surgical removal of third molar and germectomy, objectively and subjectively.

Material and Methods: A prospective nonrandomized study for all patients who were undergoing minor oral surgery of mandibular third molar was carried out in the Oral \& Maxillofacial Department (OMF) of Sultan Abdul Halim Hospital, Malaysia (HSAH). The indication for surgery was for orthodontic reasons. The patients were divided into three groups according to their radiographic root morphology, namely Group I (root not formed), Group II (root partially formed), and Group III (roots fully formed) Results: A total of 44 patients were enrolled into this study; 2 teeth from each patient, henceforth involving 88 teeth. There was a statistically significant difference in the age between the three groups $(p<0.05)$. Germectomy was the shortest procedure. In the objective evaluation conducted 1 week post-surgery, no sign of trismus and facial swelling was observed in these three groups. In the subjective evaluation, we found there were significant differences in patients' ability to tolerate orally $(p<0.05)$ and ability to perform daily activities $(p<0.05)$ among the three groups.

Conclusion: This study shows low incidence of complications in all the three groups. Germectomy is a simple and safe procedure; thus, this prompted the author advocate the early removal of mandibular third molar.
Department of Oral \& Maxillofacial Surgery, Sultan Abdul Halim Hospital, Sungai Petani, Kedah, Malaysia

*Corresponding to: Xiao F. Ling, Department of Oral \& Maxillofacial Surgery, Sultan Abdul Halim Hospital, Sungai Petani, Kedah, Malaysia drxfling@hotmail.com

Received: 15 September 2017 Revised: 21 September 2017 Accepted: 30 October 2017 Available online: 1 December 2017

Keywords: Germectomy, Mandibular Third Molar, Surgical Removal Cite this Article: Ling XF, Heng TL, Mohamad AFB. 2017. Early removal of asymptomatic mandibular third molar-is it safe? Journal of Dentomaxillofacial Science 2(3): 172-175. D0I: 10.15562/jdmfs.v2i3.611

\section{Introduction}

The mandibular third molar, which is the lower wisdom tooth, has the highest rate of impaction as compared to all the teeth, primarily due to the lack of alveolar arch space between the anterior border of ramus and the distal surface of the second molar. ${ }^{1,2}$ Surgical removal of mandibular third molar, including germectomy for orthodontic purposes, is one of the most common procedures in our facility. Germectomy is a procedure of removing a tooth that has formed one-third or less of its root and has a radiographically discernible periodontal ligament. ${ }^{3}$

Early removal of these teeth is widely acknowledged, as allegedly many studies have demonstrated a direct correlation between age and the incidence of complications. ${ }^{4}$ However, there is still lack of clinical evidence in texts, whereby the duration of the procedure and postoperative sequelae between germectomy and surgical removal of third molar were compared for further analysis. The purpose of this study was to compare the incidence of complications in relation to germectomy, early removal of third molar and surgical removal of third molar, objectively as well as subjectively.

\section{Material and methods}

For the purpose this study, a prospective non-randomized study for all patients undergoing minor oral surgery of mandibular third molar was carried out in Oral and Maxillofacial Department (OMF), Sultan Abdul Halim Hospital(HSAH), from April to September 2014. The patients were divided into three groups according to their radiographic root morphology: Group I (roots not formed), Group II (roots partially formed) and Group III (roots fully formed) figures 1-3. The study had followed the tenets of the Helsinki Declaration on medical protocol and ethics for research involving human subjects and informed consent was obtained from all participants and approved by the National Ethical Committee (Medical Research and Ethics Committee). This research did not receive any specific grant from funding agencies in the public, commercial, or not-forprofit sectors. To be included in the study sample, all patients aged between 14 and 23 years must have bilateral mandibular third molar. The patients are excluded from the study if they have a history of acute peri-coronitis; pregnancy; underlying medical illnesses such as metabolic disorder, cardiac problems 


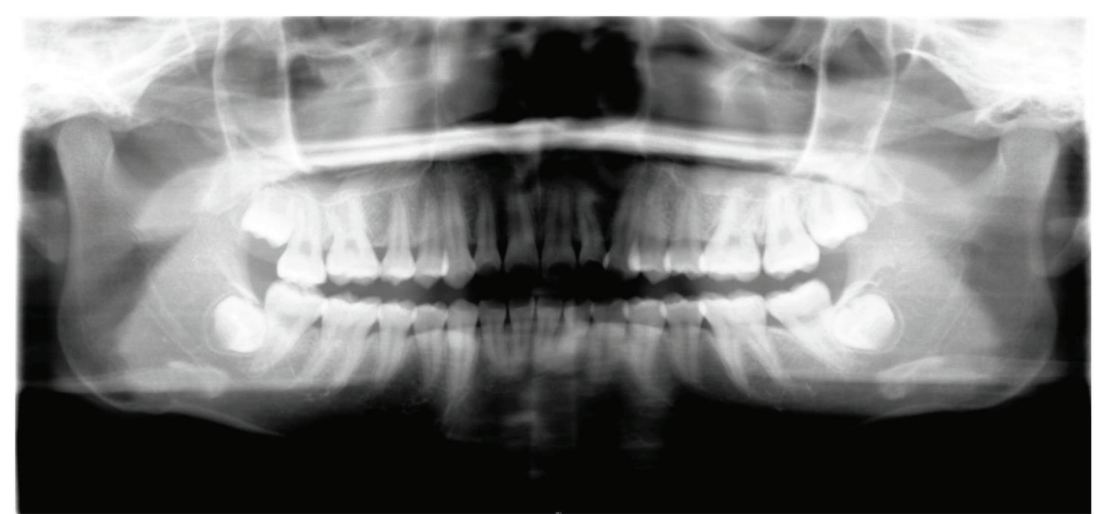

Figure 1 Panoramic radiograph showing left and right mandibular third molars with no roots formed; indicated for germectomy

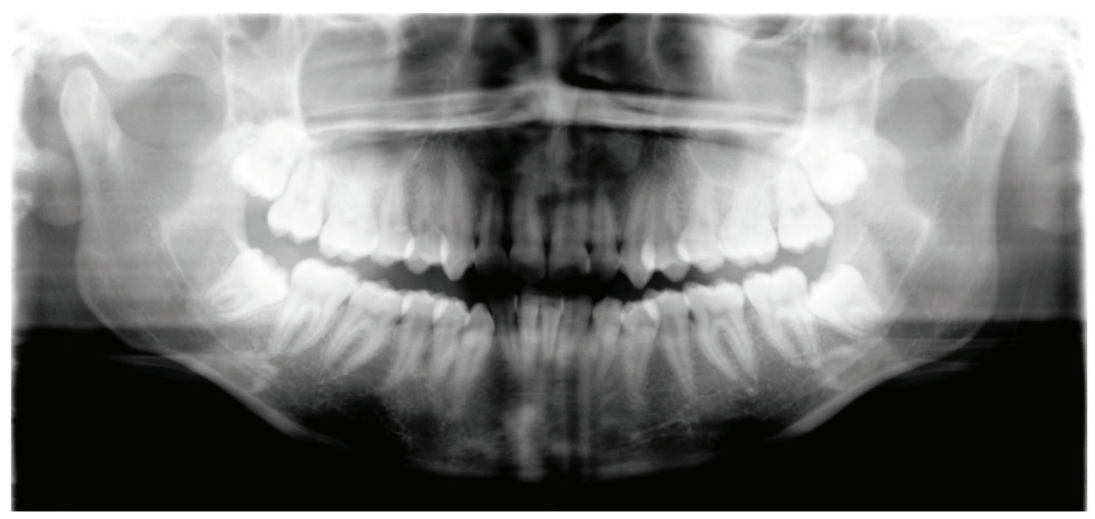

Figure 2 Panoramic radiograph showing left and right mandibular third molars with roots partially formed, indicated for early removal

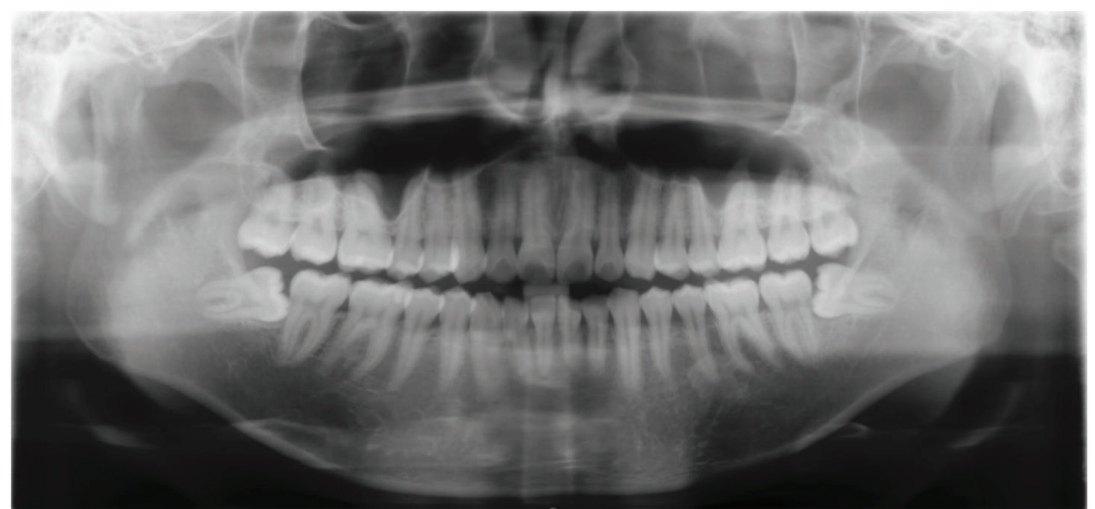

Figure 3 Panoramic radiograph showing left and right mandibular third molars with roots fully formed and closed apex; indicated for surgicalremoval

and liver or kidney diseases; bleeding disorder; and immunocompromised patients such as those affected by acquired immunodeficiency syndrome (AIDS) and psychosis. The removal of mandibular third molar was performed under general anesthesia by the same surgeon, using the standardized surgical protocol and postoperative management. Preoperatively, demographic data and baseline clinical data were recorded. Informed consents were obtained from patients preoperatively in
OMF, HSAH. For all patients, buccal incision was performed and three-sided mucoperiosteal flap was raised. Alveolar bone was removed with round burs (Meisinger HM1T 023; Meisinger, Neuss, Germany) in order to expose the tooth. Crown was sectioned with fissure burs (Meisinger HM33T 016; Meisinger, Neuss, Germany) and elevated in total. Roots were elevated in total in Groups II and III. Debridement and irrigation were performed and primary closure was done on surgical site using Vicryl 4/0 (Vicryl Ethicon, USA). The duration of surgery was defined as the time starting from first incision to the time of the last suture placed, and it was recorded in minutes. Patients from all groups were given the same oral antibiotics and analgesics: capsule amoxicillin $500 \mathrm{mg}$ 8hourly (Hovid, Malaysia) and tablet diclofenac sodium (Voltaren, Unique Pharmaceutical Labs, India) $50 \mathrm{mg}$ 8-hourly for 1 week. The patients were evaluated post-operatively 1 week later, and any postoperative complications were managed and recorded.

\section{Statistical analysis}

All data were expressed as the mean value \pm standard deviation of the mean. The method of ANOVA with post hoc Bonferroni's test was used for making multiple comparisons among the three groups. The Pearson chi square test was used to determine differences between the groups in terms of the incidence of inferior alveolar nerve deficit, presence of infection, facial swelling, and trismus. Probability of less than 0.05 was considered significant. The statistical analysis was conducted using the Statistical Package for Social Sciences for Windows, version 14.0 (SPSS, Chicago IL).

\section{Results}

A total of 44 patients were enrolled into this study, 2 teeth for each patient, and therefore involving 88 teeth. The clinical and demographic characteristics of the 44 patients are listed in table 1 . There was

\begin{tabular}{lcc} 
Table 1 & Demographic data & \\
\hline Variable & Mean \pm SD & $\mathbf{n}(\%)$ \\
\hline Age (Years) & $18.6 \pm 2.52$ & \\
Gender & & \\
$\quad$ Male & $11(25.0)$ \\
$\quad$ Female & & $33(75.0)$ \\
Group & \\
$\quad$ I & $22(25.0)$ \\
II & $30(34.1)$ \\
III & $36(40.9)$ \\
\hline
\end{tabular}

Note: $\mathrm{SD}=$ standard deviation 
Table 2 Age of patients

\begin{tabular}{ccc}
\hline \multirow{2}{*}{ Variable } & Age (years) & \\
\cline { 2 - 2 } Group & Mean \pm SD & \\
I & & \\
II & $16.1 \pm 1.48$ & $<0.001$ \\
III & $18.1 \pm 2.26$ & \\
\hline
\end{tabular}

Note: Post hoc test, Group I vs II ( $\mathrm{p}<0.001)$, II vs III $(\mathrm{p}<0.001)$, I vs III $(\mathrm{P}<0.001)$

$\mathrm{SD}=$ Standard Deviation

${ }^{\mathrm{a}}=$ One Way Anova Test

\section{Table 3 Operating time}

\begin{tabular}{lcc}
\hline \multirow{2}{*}{ Variable } & Operating time (Minutes) & \\
\cline { 2 - 3 } Group & Mean \pm SD & \\
I & & \\
II & $14.8 \pm 3.89$ & 0.007 \\
III & $15.1 \pm 6.33$ & \\
\hline
\end{tabular}

Note: Post hoc test, Group II vs III ( $\mathrm{p}=0.021)$, I vs III ( $\mathrm{P}=0.024)$

$\mathrm{SD}=$ Standard Deviation

${ }^{\mathrm{a}}=$ One Way Anova Test

Table 4 Post operative objective evaluations

\begin{tabular}{|c|c|c|c|c|c|c|}
\hline & \multicolumn{2}{|c|}{ IAN Deficit } & \multirow{3}{*}{ p-value a } & \multicolumn{2}{|c|}{ Infection } & \multirow[b]{3}{*}{ p-value a } \\
\hline & Yes & No & & Yes & No & \\
\hline & $n(\%)$ & $n(\%)$ & & $n(\%)$ & $n(\%)$ & \\
\hline \multicolumn{7}{|c|}{ Group } \\
\hline I & $0(0.0)$ & $22(100.0)$ & \multirow{3}{*}{0.482} & $1(4.5)$ & $21(95.5)$ & \multirow{3}{*}{0.219} \\
\hline II & $0(0.0)$ & $30(100.0)$ & & $0(0.0)$ & $30(100.0)$ & \\
\hline III & $1(2.8)$ & 35 (97.2) & & $0(0.0)$ & $36(100.0)$ & \\
\hline
\end{tabular}

Note: ${ }^{a}=$ Pearson chi square, IAN: Inferior Alveolar Nerve

Table 5 Post operative subjective evaluations

\begin{tabular}{|c|c|c|c|c|c|c|}
\hline & \multicolumn{2}{|c|}{$\begin{array}{c}\text { Able to Tolerate } \\
\text { orally }\end{array}$} & \multirow{3}{*}{ p-value a } & \multicolumn{2}{|c|}{$\begin{array}{l}\text { Daily Activities } \\
\text { Affected }\end{array}$} & \multirow[b]{3}{*}{ p-value ${ }^{a}$} \\
\hline & Yes & No & & Yes & No & \\
\hline & $n(\%)$ & $n(\%)$ & & $n(\%)$ & n(\%) & \\
\hline \multicolumn{7}{|c|}{ Group } \\
\hline I & $20(90.1)$ & $2(9.1)$ & & $2(9.1)$ & $20(90.1)$ & \multirow{3}{*}{0.017} \\
\hline II & $18(60.0)$ & $12(40.0)$ & 0.023 & $0(0.0)$ & $\begin{array}{c}30 \\
(100.0)\end{array}$ & \\
\hline III & $21(58.3)$ & $15(41.7)$ & & $8(22.2)$ & $28(77.8)$ & \\
\hline
\end{tabular}

Note: ${ }^{\mathrm{a}}=$ Pearson chi square

a statistically significant difference in age among the three groups $(p<0.05)$ table 2 . Germectomy was the shortest procedure. Mean operating time for Group I was shorter than that spent for Group III, and the differences were statistically significant $(p<$ $0.05)$. However, there is no statistically significant difference between Group I and Group II ( $p>0.05)$ table 3. In the objective evaluation postoperatively 1 week later, no sign of trismus and facial swelling was observed in all the three groups. One case in Group I developed postoperative infection, and one case in Group III developed transient inferior alveolar nerve deficit table 4 . In the subjective postoperative evaluation conducted 1 week later, we found there were significant differences in patients' ability in tolerating orally $(p<0.05)$ and ability to perform daily activities $(p<0.05)$ in all the three groups table 5 .

\section{Discussion}

Tooth germ of mandibular third molar was radiographically visible at an average age of 9.8 years (range 7.5-12.2 years), while the crown and half of the root was formed around 16.3 years of age (range 10.5-18.1 years). ${ }^{4}$ The subjects for this study were mainly undergoing orthodontic treatment in which the early removal of mandibular third molar might enhance the outcome of orthodontic treatment (uprighting the second molar, non-extraction orthodontic treatment, relieving of posterior crowding and prior to orthognathic surgery) and improved dental stability. Duration of procedure was significantly shorter in the Group I compared to Group II and Group III. Generally, the difficulty in the removal of mandibular third molar increased with age. ${ }^{8}$ Study showed aging would cause the mandible to be sclerotic; moreover, tendency of hypercementosis and ankyloses of a tooth increased with age. ${ }^{9}$ Incidence of early postoperative complications was low in these groups. Nonetheless, the incidence of transient inferior alveolar nerve injury was $1.13 \%$ in Group III. This may be due to stretching, heat generated during burring, or local anesthesia effect on the inferior alveolar nerve. ${ }^{10}$ Some suggested that it might be due to the intratooth pressure transmitted to the nerve during the process of crown splitting. ${ }^{11}$ Postoperatively, patient was prescribed with tablets vitamin B1, B6, and B12 (vitbion forte, dynapham, Malaysia). However, tactile sensitivity test and pain test showed positive results after one month. On the other hand, the incidence of early postoperative infection was $1.13 \%$ in Group I. This was due to poor oral hygiene. The infection was resolved after a course of oral antibiotics, local debridement, and reinforcement of oral hygiene instructions. The overall result revealed that all patients experienced no facial swelling 1 week postoperatively. This was in line with the findings of other studies: maximum 
age of 25 years. Studies have shown that complications with surgical removal of mandibular third molar increased over the age of 25 years. $^{9}$ The complication rate was 1.5 times to 3 times greater in patients aged over 25 years than in those aged below 25 years., ${ }^{4,15}$ Impacted third molar may cause external root resorption of a second molar. Resorption was correlated with age and mesioangular impaction. Our study found that $2.4 \%$ of the mandibular second molars showed significant resorption caused by non-erupted mandibular third molars. ${ }^{16}$ Impacted mandibular third molar might also cause large periodontal defects of mandibular second molar. These periodontal defects would heal if the mandibular third molar was removed at a younger age as compared to delayed removal. ${ }^{1}$ Delaying the removal of impacted mandibular third molars until a problem arises will subject the patient to unnecessary pain, infection, and more complex and costly operations with higher risk of complications during surgery and postoperative phases. ${ }^{4,17,18}$ In order to minimize bias in this study, we followed the study protocol strictly and all surgeries were performed by the same oral maxillofacial surgeon who is the first author.

\section{Conclusion}

This study shows low incidence of complications in all the three groups. The delayed removal of impacted mandibular third molars until a problem arises will subject the patient to unnecessary pain and complex operations, not to mention the higher risk of postoperative complications. Germectomy is a simple and safe procedure; thus, this prompted the author advocate the early removal of mandibular third molar.

\section{Acknowledgments}

Director General of Health Malaysia for permission to publish this research. We would like to acknowledge Miss Chanel Chau Xue Rou and Miss Tew Mei Mei who have made significant contributions to this study.

\section{Conflict of Interest}

The authors report no conflict of interest.
2. Beeman CS. Third molar management: a case for routine removal in adolescent and young adult orthodontic patients. J Oral Maxillofac Surg 1999;57: 824-830.

3. Sivolella S, Berengo M, Bressan E, et al. Osteotomy for lower third molar germectomy: randomized prospective crossover clinical study comparing piezosurgery and conventional rotatory osteotomy. J Oral Maxillofac Surg 2011;69: e15-23.

4. Chiapasco M, Crescentini M, Romanoni G. Germectomy or delayed removal of mandibular impacted third molars: the relationship between age and incidence of complications. J Oral Maxillofac Surg 1995;53: 418-433.

5. Bui CH, Seldin EB, Dodson TB. Types, frequencies, and risk factors for complications after third molar extraction. J Oral Maxillofac Surg 2003;61: 1379-1389.

6. Sisk AL, Hammer WB, Shelton DW, et al. Complications following removal of impacted third molars: the role of the experience of the surgeon. J Oral Maxillofac Surg 1986;44: 855-859.

7. Blondeau F, Daniel NG. Extraction of impacted mandibular third molars: postoperative complications and their risk factors. J Can Dent Assoc 2007;73: 325.

8. Bouloux GF, Steed MB, Perciaccante VJ. Complications of third molar surgery. Oral Maxillofac Surg Clin North Am 2007;19: 117-128.

9. Pogrel MA. What is the effect of timing of removal on the incidence and severity of complications? J Oral Maxillofac Surg 2012;70: S37-40.

10. Chossegros C, Guyot L, Cheynet F, et al. Is lingual nerve protection necessary for lower third molar germectomy? a prospective study of 300 procedures. Int J Oral Maxillofac Surg 2002;31: 620-624.

11. Knutsson K, Lysell L, Rohlin M. Postoperative status after partial removal of the mandibular third molar. Swed Dent J 1989;13: 15-22.

12. Chukwuneke FN, Oji C, Saheeb DB. A comparative study of the effect of using a rubber drain on postoperative discomfort following lower third molar surgery. Int J Oral Maxillofac Surg 2008;37: 341-344.

13. UStün Y, Erdogan O, Esen E, et al. Comparison of the effects of 2 doses of methylprednisolone on pain, swelling, and trismus after third molar surgery. Oral Surg Oral Med Oral Pathol Oral Radiol Endod 2003;96: 535-559.

14. Cerqueira PRF, Vasconcelos BC do E, Bessa-Nogueira RV. Comparative study of the effect of a tube drain in impacted lower third molar surgery. J Oral Maxillofac Surg 2004;62: 57-61.

15. Chuang S-K, Perrott DH, Susarla SM, et al. Age as a risk factor for third molar surgery complications. J Oral Maxillofac Surg 2007;65: 1685-1692.

16. Nemcovsky CE, Libfeld H, Zubery Y. Effect of non-erupted 3rd molars on distal roots and supporting structures of approximal teeth. A radiographic survey of 202 cases. J Clin Periodontol 1996;23: 810-815.

17. Jones ML, Armstrong R, Kostopoulou O, et al. Treatment strategies for lower third molars following orthodontic care. Br J Orthod 1997;24: 319-324.

18. Leone SA, Edenfield MJ, Cohen ME. Correlation of acute pericoronitis and the position of the mandibular third molar. Oral Surg Oral Med Oral Pathol 1986;62: 245-250.

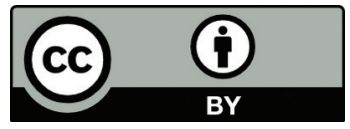

This work is licensed under a Creative Commons Attribution

1. Waite PD, Reynolds RR. Surgical management of impacted third molars. Semin Orthod 1998;4: 113-123. 